\title{
ROLE OF ULTRASOUND IN EVALUATION OF NON-TRAUMATIC SHOULDER PAIN
}

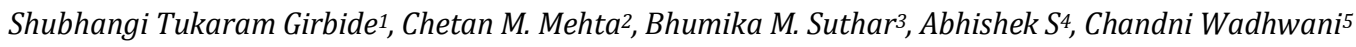

${ }_{1}^{1}$ Resident, Department of Radio-Diagnosis, Baroda Medical College and SSC-Hospital, Vadodara, Gujarat. ${ }^{2}$ Associate Professor, Department of Radio-Diagnosis, Baroda Medical College and SSC-Hospital, Vadodara, Gujarat. ${ }^{3}$ Assistant Professor, Department of Radio-Diagnosis, Baroda Medical College and SSC- Hospital, Vadodara, Gujarat. ${ }^{4}$ Resident, Department of Radio-Diagnosis, Baroda Medical College and SSC-Hospital, Vadodara, Gujarat. ${ }^{5}$ Resident, Department of Radio-Diagnosis, Baroda Medical College and SSC-Hospital, Vadodara, Gujarat.

ABSTRACT
BACKGROUND
Shoulder is one of the anatomical areas which is most commonly evaluated with musculoskeletal ultrasonography.
The objectives of this study were-
1. To evaluate the role of ultrasound in non-traumatic shoulder pain.
2. Diagnose different rotator cuff and non-rotator cuff pathologies of shoulder with the help of ultrasonography.

\section{MATERIALS AND METHODS:}

This prospective observational study was done in department of Radiodiagnosis, Baroda Medical College and SSG Hospital, Vadodara, Gujarat on the patients who presented with history of shoulder pain. A total of 80 patients was subjected to high resolution ultrasonography between January 2017 to October 2017 for period of 9 months. It was performed by using Phillips iU22 and My lab 20 machines, with a $10 \mathrm{MHz}$ frequency linear transducer. Colour Doppler was used to evaluate for the vascularity of the lesion.

\section{RESULTS}

Total 80 patients had been subjected to ultrasonography, out of which 51 patients were having some shoulder pathology and 29 patients were sonographically normal.

Rotator cuff tear was the commonest pathology found followed by bicep tendinosis, acromioclavicular joint arthropathy, calcific tendinopathy, subacromial-subdeltoid bursitis, Hill Sach's lesion, glenohumeral joint effusion, synovitis and osteosarcoma.

\section{CONCLUSION}

Ultrasonography is cheapest modality of choice which is able to diagnose different rotator and non-rotator cuff pathologies of shoulder.

\section{KEYWORDS}

Ultrasonography, Rotator Cuff.

HOW TO CITE THIS ARTICLE: Girbide ST, Mehta CM, Suthar BM, et al. Role of ultrasound in evaluation of non- traumatic shoulder pain. J. Evolution Med. Dent. Sci. 2018;7(09):1157-1161, DOI: 10.14260/jemds/2018/263

\section{BACKGROUND}

Shoulder is one of the anatomical areas that is most commonly evaluated with musculoskeletal ultrasonography. When performed with appropriate equipment, by skilled operators, Ultrasound is widely recognized as a means of accurately assessing rotator cuff disease, with a sensitivity and specificity as high as $90 \%-95 \%$ in the assessment of both partial- and full-thickness tears. ${ }^{1,2}$

Shoulder can be very easily assessed because of anatomical reasons. It is an inexpensive modality. Knowledge of optimal techniques, normal anatomy, dynamic manoeuvres, and pathological conditions is essential for correct US imaging and interpretation. ${ }^{3}$ Comparison between the damaged and the contralateral sides may aid in reaching diagnostic conclusions. ${ }^{4}$

'Financial or Other Competing Interest': None.

Submission 01-01-2018, Peer Review 08-02-2018,

Acceptance 14-02-2018, Published 26-02-2018.

Corresponding Author:

Dr. Bhumika M. Suthar,

201, Vivanta Classic Flats,

Behind Soham Bungalows,

Vasna Road, Near Manisha Chokdi,

Vadodara, Gujarat.

E-mail: bhums22@yahoo.co.in

DOI: $10.14260 /$ jemds $/ 2018 / 263$
Different pathologies like rotator cuff tears, calcific tendinopathy, bicep tendinosis, shoulder joint effusion, subacromial-subdeltoid bursitis, acromioclavicular joint arthropathy, bicep tendon tear, synovitis can be diagnosed with the help of ultrasonography. This study was done to know the different rotator and non-rotator cuff pathologies of shoulder.

\section{MATERIALS AND METHODS}

This prospective observational study was done on 80 patients for period of 9 months from January 2017 to October 2017. Majority of patients were between 35 to 50 yrs. Out of 80 patients 62 males and 18 females. Data was collected by simple random technique and referred to radiology department for ultrasonography. High resolution ultrasound was performed using $10 \mathrm{mHz}$ linear transducer of Phillips iU22 and My lab 20 machines. Ultrasound was performed both in static and dynamic manoeuvres. Patients who were having shoulder pain with no history of trauma were included in this study.

\section{Aims and Objectives}

1. To evaluate the role of ultrasound in non-traumatic shoulder pain. 
2. Diagnose different rotator cuff and non-rotator cuff pathologies of shoulder with the help of ultrasonography.

\section{Statistics}

High resolution ultrasonography of shoulder was performed on 80 patients. Male patients were more than female patients.

\begin{tabular}{|c|c|c|}
\hline Sex & Number of Patients & Percentage \\
\hline Male & 62 & $77.50 \%$ \\
\hline Female & 18 & $22.50 \%$ \\
\hline Total & $\mathbf{8 0}$ & $\mathbf{1 0 0 . 0 0 \%}$ \\
\hline \multicolumn{3}{|c|}{ Table 1. Sex distribution } \\
\hline
\end{tabular}

Maximum Number of patients were in 36 to 50 years age group.

\begin{tabular}{|c|c|c|}
\hline Age Distribution & Number of Patients & Percentage \\
\hline 20 to 35 & 20 & $25.00 \%$ \\
\hline 36 to 50 & 50 & $62.50 \%$ \\
\hline 51 to 65 & 10 & $12.50 \%$ \\
\hline Total & $\mathbf{8 0}$ & $\mathbf{1 0 0 . 0 0 \%}$ \\
\hline \multicolumn{2}{|c|}{ Table 2. Age distribution } \\
\hline
\end{tabular}

Right shoulder (88.75\%) involvement was more common than left shoulder (11.25\%).

\begin{tabular}{|c|c|c|}
\hline Shoulder & Number of Patients & Percentage \\
\hline Right & 71 & $88.75 \%$ \\
\hline Left & 9 & $11.25 \%$ \\
\hline Total & $\mathbf{8 0}$ & $\mathbf{1 0 0 . 0 0 \%}$ \\
\hline Table 3. Distribution as per shoulder involvement \\
\hline
\end{tabular}

The most common cause of non-traumatic shoulder pain was rotator cuff tear was seen in 17 patients $(21.25 \%)$.

\begin{tabular}{|c|c|c|}
\hline Rotator Cuff Tendon Tear & No. of Patients & $\%$ \\
\hline Supraspinatus & 16 & $20.00 \%$ \\
\hline Subscapularis & 3 & $3.75 \%$ \\
\hline Infraspinatus & 1 & $1.25 \%$ \\
\hline Teres minor & 0 & $0 \%$ \\
\hline
\end{tabular}

\begin{tabular}{|c|c|c|}
\hline Pathology & No. of Patients & Percentage \\
\hline Rotator cuff tear & 17 & $21.25 \%$ \\
\hline Bicep tendinosis & 7 & $8.75 \%$ \\
\hline AC joint & 6 & $7.50 \%$ \\
\hline Calcific Tendinopathy & 5 & $6.25 \%$ \\
\hline SA-SD bursitis & 5 & $6.25 \%$ \\
\hline Hill Sachs lesion & 4 & $5.00 \%$ \\
\hline Joint effusion & 3 & $3.75 \%$ \\
\hline Synovitis & 2 & $2.50 \%$ \\
\hline Bicep tendon tear & 1 & $1.25 \%$ \\
\hline Osteosarcoma & 1 & $1.25 \%$ \\
\hline Normal & 29 & $36.25 \%$ \\
\hline Total & $\mathbf{8 0}$ & $\mathbf{1 0 0 . 0 0} \%$ \\
\hline Table 5. Distribution as per shoulder pathology \\
\hline \multicolumn{2}{|c}{}
\end{tabular}

\section{RESULTS}

Out of 80 patients, $62(77.5 \%)$ patients were male and 18 patients were female (22.5\%). Right shoulder involvement was more common than left shoulder in both male as well as female. Right shoulder involvement was seen in 71 patients (88.75\%) and left shoulder in 9 patients $(11.25 \%)$.

Maximum number of patients were in 36 to 50 years age group $(62.5 \%)$ and minimum number of patients were in 51 to 65 years $(12.5 \%)$ age group.

Out of 80 patients, 51 patients $(63.75 \%)$ were having some shoulder pathology and 29 patients (36.25\%) were having normal sonography. Most common pathology responsible for shoulder pain was rotator cuff tear which was seen in 17 patients (21.5\%). High resolution ultrasonography helps in differentiating full thickness and partial thickness tear. In both the cases, supraspinatus was most commonly involved tendon seen in 16 cases (20\%). Second most common pathology was bicep tendinosis seen in 7 patients (8.75\%), followed by acromioclavicular joint arthropathy seen in 6 patients $(7.25 \%)$.

There were 5 patients $(6.25 \%)$ of calcific tendinopathy. Supraspinatus was most commonly involved tendon. 5 patients (6.25\%) of isolated subacromial subdeltoid bursitis, without any obvious underlying rotator cuff tear. Hill Sachs lesion was seen in 4 patients (5\%) and glenohumeral joint effusion was seen in 3 patients (3.75\%).There were 2 patients of synovitis (2.5\%). There was one patient $(1.25 \%)$ of bicep tendon tear and osteosarcoma each.

Out of 80 patients 29 patients (36.25\%) were having normal sonography. These patients should not be interpreted as normal because ultrasound is not definitive investigation of choice. MRI is the gold standard.
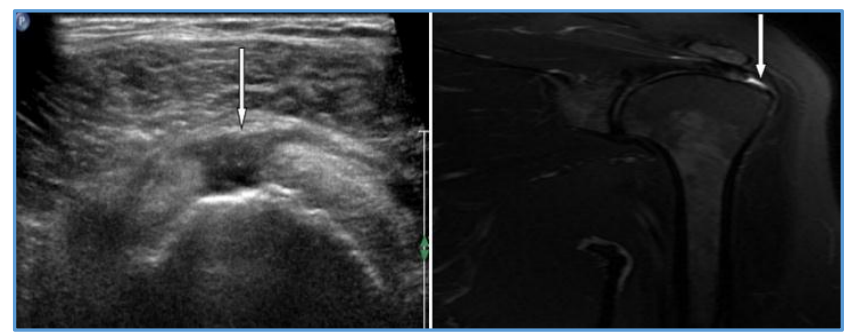

Figure 1a, 1b: Full-thickness supraspinatus tear. Short axis view of supraspinatus tendon shows an obvious focal area of discontinuity (arrow) involving supraspinatus tendon. Magnetic resonance imaging of the same patient, Coronal T2 fat saturated image, showing with full thickness tear of supraspinatus with retraction of fibres

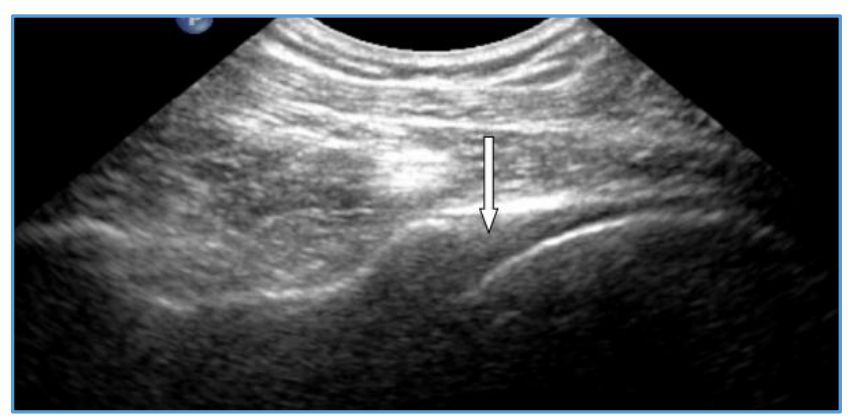

Figure 2a. Infraspinatus tear, posterior aspect of shoulder shows non-visualisation of echogenic tendon of infraspinatus suggestive of tear 


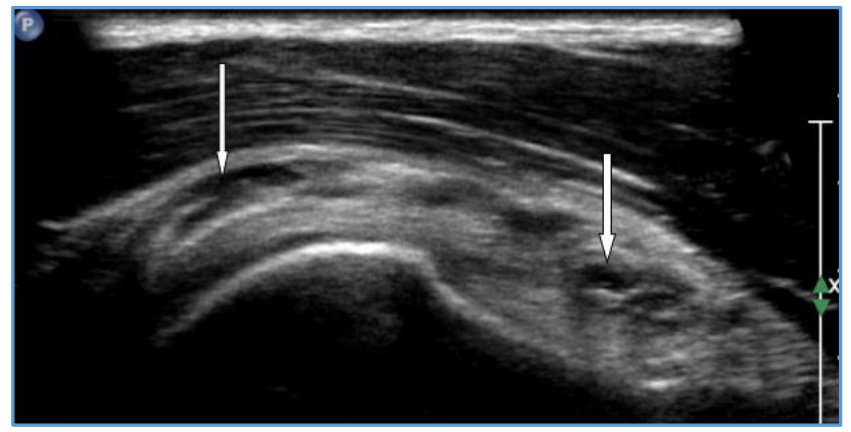

Figure 3. Partial-thickness tear of the supraspinatus. Long axis view of the supraspinatus tendon; showing focal discontinuity of fibers of supraspinatus tendon (arrows)

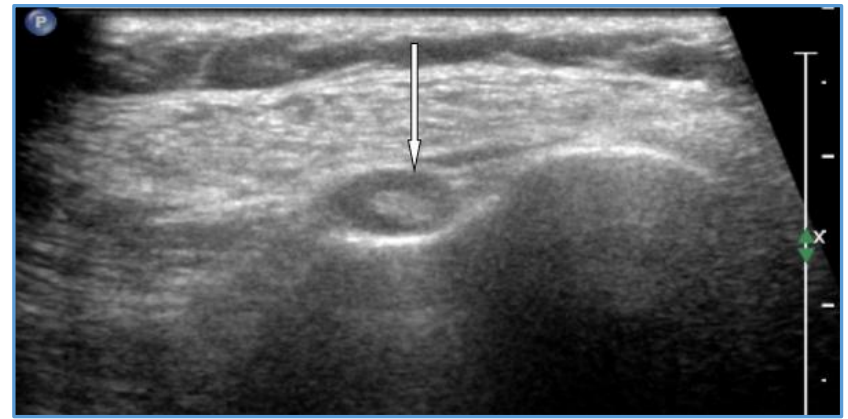

Figure 4. Bicep tendinosis; Short axis view of bicep tendon in bicipital groove, showing the anechoic fluid collection surrounding the bicep tendon
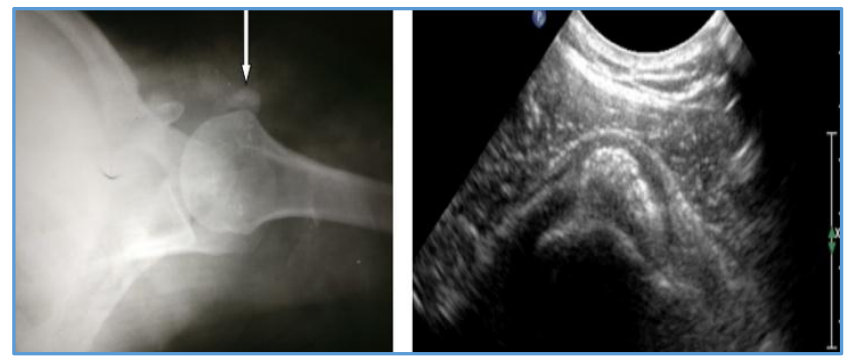

Figure 5a, 5b. Calcific tendinopathy; Axillary view X-ray left shoulder, showing the abnormal calcification(arrow) noted in relation to greater tubercle of humerus and long axis view of the supraspinatus tendon showing the calcification (arrow) within the supraspinatus tendon

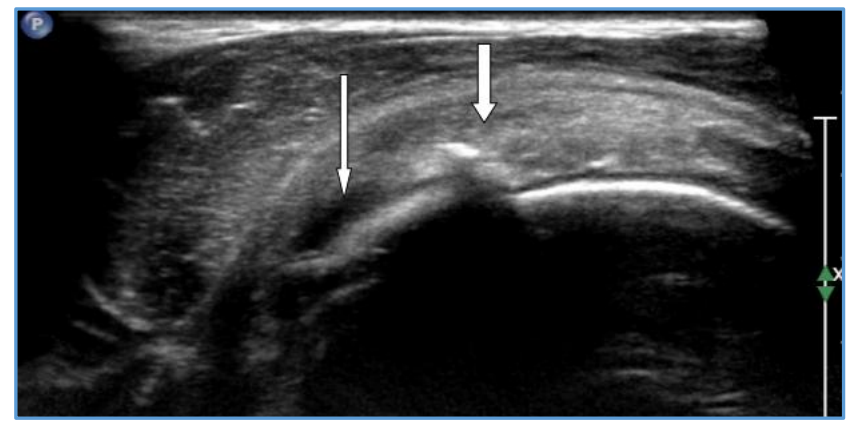

Figure 5c. Calcific tendinopathy. Short axis view of the supraspinatus tendon shows tiny calcification (Thick arrow) and also shows partial thickness tear at the site of insertion (Thin arrow)
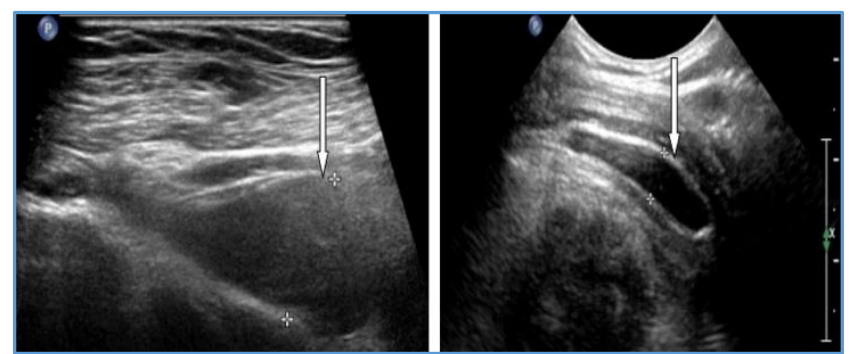

Figure 6a, 6b. Subacromial subdeltoid bursitis, image showing the gross distention of bursa with anechoic fluid (Arrow)

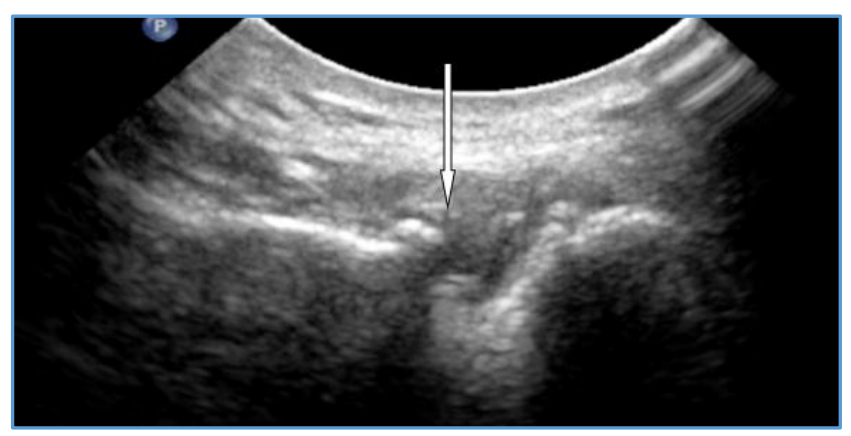

Figure 7. Acromioclavicular joint arthropathy, showing cortical surface irregularity, joint space reduction with marginal osteophytes (Arrow)

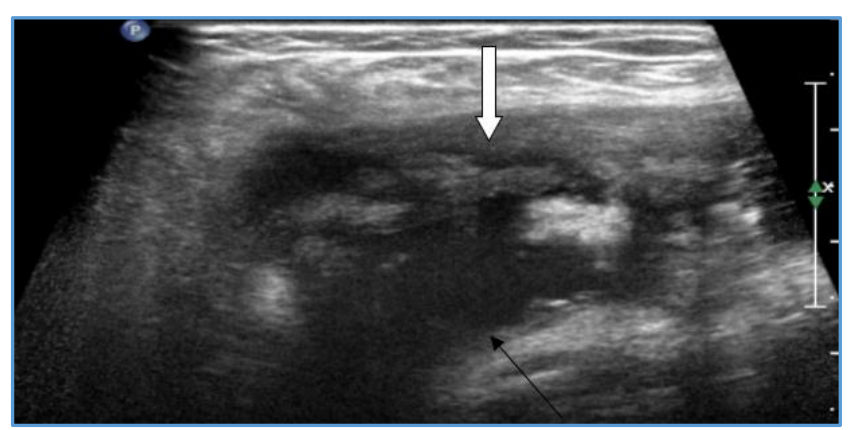

Figure 8. Synovitis with joint effusion; shows proliferation of hypoechoic synovium (Thick arrow) with joint effusion (Thin arrow) with destruction of underlying rotator cuff

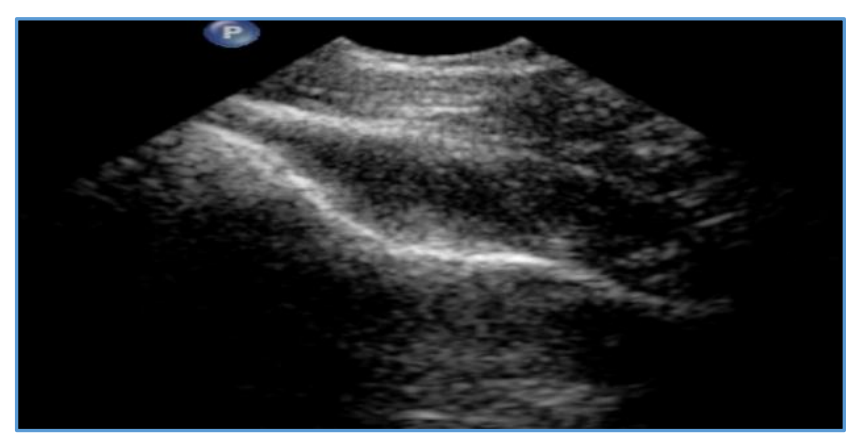

Figure 9. Hill Sachs lesion, bony depression over posterolateral aspect of head of humerus 

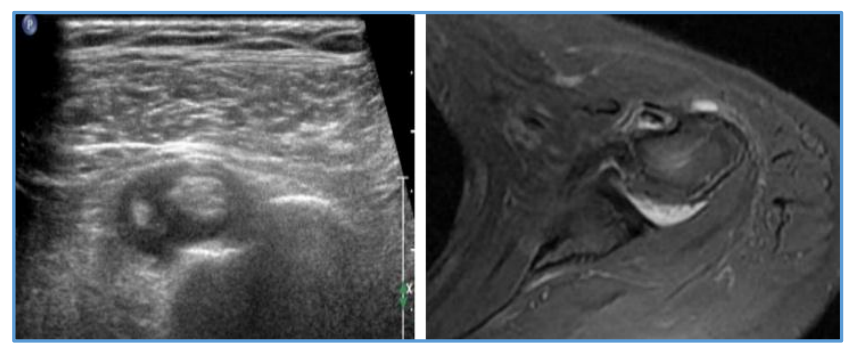

Figure 10 a. Bicep tendon tear, short axis view vertical tear in tendon of long head of bicep in bicipital groove. Magnetic resonance imaging of same patient, axial proton density image of shoulder, shows hyperintense signal intensity within in long head of bicep tendon with surrounding fluid in bicipital groove
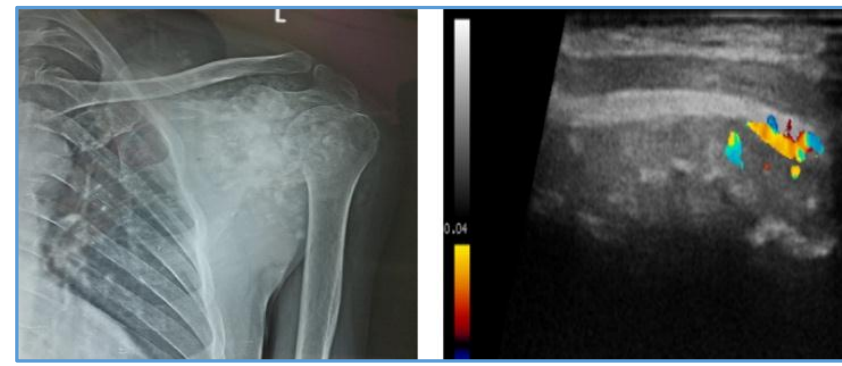

Figure 11 a, 11 b. Anteroposterior view of X-ray left shoulder, shows abnormal cumulus cloud like calcification noted in relation to scapula and shoulder joint. Ultrasound of anterior aspect of left shoulder, Showing ill-defined hypoechoic lesion with internal vascularity within, with multiple calcific foci giving after shadow, causing destruction of underlying rotator cuff

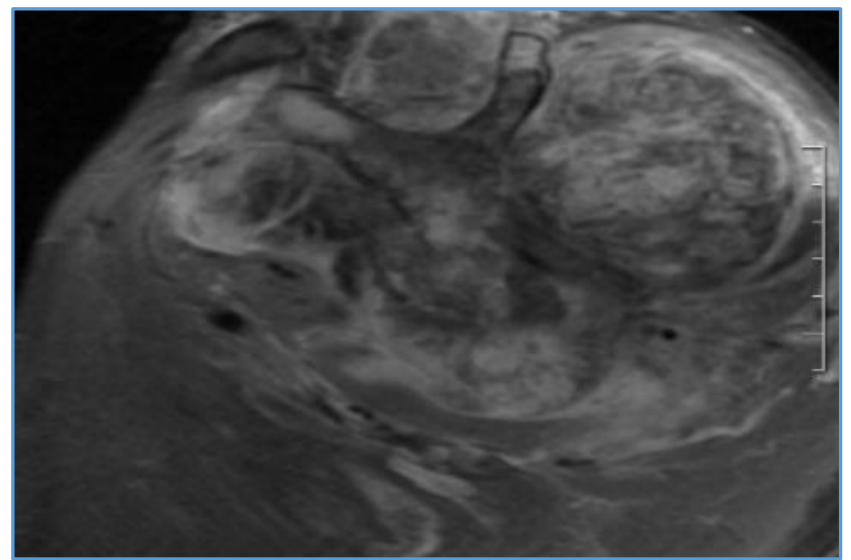

Figure 11 c. Biopsy proven osteosarcoma of left shoulder, Sagittal proton density image showing large

heterogeneously hyperintense lesion with multiple hypointense areas within, causing destruction of rotator cuff muscles and scapula

\section{DISCUSSION}

As per Pradeep Goyal et $\mathrm{al}^{5}$ ultrasound showed supraspinatus lesion in 21(\%) of painful shoulder and least commonly involved tendon was teres minor. This correlated with our study in which supraspinatus was involved in $(21.25$ $\%$ ) and least commonly involved tendon was teres minor with no case evidence.

In this study, rotator cuff tear was the main pathology $(22.5 \%)$ responsible for shoulder pain. Tear appears as discontinuity in fibres of tendon with anechoic fluid collection in the gap. Tear is of two types full (Figure.1a) and partial thickness tear (Figure.1a). In both conditions, supraspinatus tendon was most commonly involved was seen in 16 patients (20\%). Subscapularis tendon tear was seen in 3 patients $(1.25 \%)$ (Figure 3$)$. Infraspinatus tear was seen in 1 patients $(1.25 \%)$ (Figure 2a).

As per A.T. Kharat et al, 6 acute tendinitis is detected by increased fluid within synovial sheath. This can be evaluated in transverse scan. In chronic tendinitis, thickening of tendon itself without increase in synovial fluid. Tenosynovitis is inflammation of tendon sheaths. In this study, acute bicep tendinitis was seen in 7 patients $(8.75 \%)$. It was seen as anechoic fluid collection around the long head of bicep tendon in bicipital groove (Figure 4).

As per Athanasios Papatheodorou7, stated that USG is helpful in evaluating the superior aspect of AC joint. By using sagittal plane, the gap between the acromion as a window, it is possible to image the joint space, bone erosions, fluid, cysts and hypertrophic changes represents degenerative changes. Acromioclavicular joint arthropathy (Figure 7) was seen in cases and all patients were above 50 yrs. It was seen in 6 patients (7.5\%) in this study.

As per Athanasios Papatheodorou7, stated that calcific tendinopathy is common disorder caused by deposition of calcium hydroxyapatite crystals in shoulder tendons. Most common cause for this is considered as dystrophic. Most commonly involved tendon is supraspinatus which is seen at insertion site due to old tear or due to chronic degenerative changes. Although all tendons can be involved. On radiograph calcification can be seen in relation to greater (Figure 5a) and lesser tubercle. It was seen in 5 patients (6.25 \%) in our study (Figure 5b, 5c)

There were 5 patients $(6.25 \%)$ of isolated subacromial subdeltoid bursitis (Figure 6a, 6b) without any rotator cuff injury. According to Marnix Van Holsbeeck et al, ${ }^{8}$ subacromial subdeltoid bursa is irritated by chronic repetitive trauma and further leading to impingement syndrome. The integrity of the underlying rotator cuff determines the grade of impingement ranging from an intact rotator cuff with bursal haemorrhage, fibrosis and tendon oedema to a torn rotator cuff.

Hill Sachs lesion is bony depression over posterolateral aspect of head of humerus, typically seen in anterior dislocation of shoulder in young patients. It is seen as wedge shaped shallow defect of hypoechoic bony contour of humeral head at the point where the anterior portion of the infraspinatus inserts into the greater tuberosity. It was seen in 4 patients (5\%) (Figure 9).

Glenohumeral joint effusion is assessed in posterior recess. It was seen in 3 cases $(3.75 \%)$ without any associated rotator cuff abnormality. However, joint effusion can be associated with rotator cuff tear. Synovitis is the proliferation of synovium. There were 2 patients of synovitis (Figure 8).

There was one patient in which full thickness tear of supraspinatus and partial thickness tear of subscapularis tendon and tear of long head of bicep tendon. Biceps tendon tear (Figure 10a, 10b) is seen as discontinuity in tendon with anechoic fluid collection in bicipital groove.

There was one patient who was having shoulder pain since 6 months. X-ray showed abnormal radio-opacity with cloud like calcification within, with destruction of scapula (Figure 11a). Ultrasonography shows hypoechoic lesion with 


\section{Jemds.com}

internal vascularity within, with multiple calcific foci within giving strong after shadow (Figure 11b) with destruction of underlying rotator cuff. On magnetic resonance imaging, lesion appears as heterogeneously hyperintense lesion with hypointense area within on proton density lesion (Figure 11c). This was histopathologically proved as case of osteosarcoma.

\section{CONCLUSION}

High resolution ultrasonography is fast, inexpensive modality be used as primary imaging modality for evaluating shoulder joint. It is efficient imaging modality for assessment of wide spectrum of rotator and non-rotator cuff pathologies. Performing the high-resolution ultrasonography of shoulder in two different planes, both in static and dynamic position, helps to eliminate the artefacts as well as helps in diagnosing different pathologies.

\section{REFERENCES}

[1] Bouffard JA, Lee SM, Dhanju J. Ultrasonography of the shoulder. Semin in Ultrasound, CT and MRI 2000;21(3):164-91.

\section{Original Research Article}

[2] Teefey SA, Middleton WD, Yamaguchi K. Shoulder sonography. State of the art. Radiol Clin North Am 1999;37(4):767-85.

[3] Le Corroller T, Cohen M, Aswad R, et al. Sonography of the painful shoulder: role of the operator's experience. Skeletal Radiol 2008;37(11):979-86.

[4] Garg P, Marathe VN, Gandage SG, et al. Ultrasonographic evaluation of painful shoulder joint in rural population. International J of Healthcare and Biomedical Research 2014;2(2).

[5] Goyal P, Hemal U, Kumar R. High resolution sonographic evaluation of painful shoulder. The Internet Journal Of Radiology 2010;12.

[6] Kharat A, Kharat K, Singh A, et al. Biceps tendon tenosynovitis-a sonologic marker of shoulder joint derangement. Indian J Radiology Imaging 2006;16(4):633-6.

[7] Papatheodorou A, Ellinas P, Takis F, et al. US of the shoulder: rotator cuff and non-rotator cuff disorders. Radiographics 2006;26(1):e23.

[8] van Holsbeeck MT, Kolowich PA, Eyler WR, et al. US depiction of partial-thickness tear of the rotator cuff. Radiology 1995;197(2):443-6. 